\title{
Consumer's Risk Perception of Genetically Modified Food and its Influencing Factors: Based on the Survey in Jiangsu Province, China
}

\author{
Ruixin $\mathrm{Liu}^{1,3}$, Linhai $\mathrm{Wu}^{1,2, *}$, Lijie Shan ${ }^{1}$ and and $\mathrm{Hua}^{2}{ }^{2}$ \\ ${ }^{I}$ Food Safety Research Base of Jiangsu Province, School of Business, Jiangnan University, Wuxi 214122, China \\ ${ }^{2}$ Synergetic Innovation Center of Food Safety and Nutrition, Wuxi 214122, China \\ ${ }^{3}$ School of Tourism, Yangzhou University, Yangzhou 225127, China
}

\begin{abstract}
Safety has always been the focus of debate on genetically modified food (GMF). To understand consumers' risk perception of GMF and its influencing factors, this study investigated 300 consumers from 6 cities in Jiangsu province, China by questionnare. The data were analyzed by using independent sample t-test, one-way ANOVA and principal component analysis. The results showed that most consumers worried about the safety of GMF and hoped GMF to be labeled with identity; meanwhile, their purchase intention of GMF was not high. Some of consumer characteristics including gender, education background, personal annual income, and with at least one child under 18, significantly influenced their risk perception of GMF. Furthermore, the main factors significantly influencing the consumers' risk perception of GMF were as follows in order of influence degree: health risks (0.386), ecological risk (0.187), and social risk (0.163). Consequently, the government should strengthen the popularization of scientific knowledge on genetically modified technology as well as GMF and simutaneously reinforce standardized management of GMF label.
\end{abstract}

Keywords: Consumers, genetically smodified food, risk perception, factor analysis.

\section{INTRODUCTION}

In recent years, with the rapid increase of urban population, continuous decrease of arable land and ever-growing material demand of people, genetically modified organism (GMO), especially genetically modified crop, has been developing fast due to its incomparable advantages such as disease and insect resistance, high yield, endurable storage, freedom from seasonal and climatic restriction, and quality improvement when compared with traditional one. Genetically modified food (GMF) is food made from GMO [1]. Because there's still no clear conclusion on GMF safety, many negative reports on GMF safety emerge constantly and GMF are even demonized on the internet. Thus, the public may resist and dread it, which has great negative influence on the promotion of genetically modified crop and the development of its relevant food processing industry.

Consumers are the direct recipients of GMF. Their consuming intention may guide market development, and factors affecting their choices may promote market transformation. According to the risk perception theory of food safety, it is not the actual risk of food safety itself but consumers' subjective risk perception of food safety that governs their consumption behavior [2]. Therefore, by making an investigation on consumers' risk perception of food safety in

*Address correspondence to this author at 2Synergetic Innovation Center of Food Safety and Nutrition, 214122, China;

Tel: +8613506179899; E-mail: wlh6799@126.com
Jiangsu Province, China, this study aimed to have an understanding of Jiangsu consumers' risk perception and attitude towards GMF, and the main factors influencing their risk perception. The results of this study may enrich the achievement of consumers' risk perception of GMF and provide theoretical support for reducing the risk. Moreover, it may provide foundation for enterprises concerning GMF to ascertain target market and make corresponding marketing strategies, thereby promoting GMF market to develop in a faster and healthier way.

\section{QUESTIONNAIRE DESIGN AND DATA ACQUISI- TION}

\subsection{Questionnaire Design}

By combining Dong's [3] measurement scale about risk perception of GMF with consumers' actual situation in Jiangsu province, we developed a questionnaire about Jiangsu consumers' risk perception of GMF and its influencing factors. The questionnaire includes three parts. The first part examines consumers' cognition and attitude to GMF; the second part investigates the main factors affecting consumers' risk perception of GMF; the third part shows consumers' individual characteristics.

\subsection{Data Acquisition}

This study employed questionnaire to collect data for its operability. Stratified and random sampling were used to ensure that the samples are universal and representative. 
First, 13 cities in Jiangsu province were divided into three regions according to their geographic location, which are southern, northern, and central Jiangsu. Then two cities in each region were selected as representatives, which are Suzhou and Wuxi, Yangzhou and Nanjing, and Huai'an and Suqian, respectively. A total of 300 questionnaires, 50 per city, were distributed. The investigation was conducted in the supermarkets, farmers' markets, and squares from April 30 to May 5, 2014. The questionnaires were completed on the spot by communicating face to face between the trained investigators and respondents. Finally, 300 questionnaires were collected. The data were analyzed by SPSS19.0.

\subsection{Characteristics of Samples}

The total of 152 males and 148 females participated in this investigation, accounting for $50.7 \%$ and $49.3 \%$ of all participants, respectively. In terms of age, 107 participants were less than 30, 132 participants between 31 and 55, and 61 participants over 55, which accounted for $35.7 \%, 44.0 \%$, and $20.3 \%$, respectively. As to education background, participants with junior high school, senior high school, and college degree accounted for $30.7 \%, 40.3 \%$, and $29.0 \%$, respectively. In regard to personal annual income in 2014, the income of 67 participants were less than 30000 yuan, that of 135 participants between 30000 and 60000 yuan, and that of 98 participants more than 60000 yuan, accounting for $22.3 \%, 45.0 \%$, and $32.7 \%$, respectively. In addition, $65.3 \%$ of the participants owned at least one child under 18 in their families.

\section{CONSUMERS' RISK PERCEPTION OF GMF}

\subsection{Cognition of GMF}

As a newborn high-tech food, GMF has become an integral part in our life in only more than 10 years. The investigation showed that $5.4 \%$ of the respondents were very familiar with GMF, $13.6 \%$ knew it, $29.0 \%$ had a little knowledge of it, $34.3 \%$ have heard of it but did not know it, and only $17.7 \%$ have never heard of it. Compared with the result of Zhong [4] who investigated Nanjing consumers' attitudes towards GMF in 2004 and found only $43.3 \%$ of the responders had heard of GMF, the percentage of the respondents having heard of GMF increased to $82.3 \%$. This increase indicates that GMF has developed rapidly in Jiangsu province, one of the most developed provinces in China, during the past 10 years.

Although $48.0 \%$ of the respondents said that they knew GMF more or less, their cognition was relatively vague in distinguishing specific GMF. They were more familiar with soybean, corn, and tomato which are common in daily life, but had a little knowledge of papaya, sweet pepper, and oilseed rape. Moreover, even $19.5 \%$ of the respondents considered non-genetically modified wheat and peanut as GMF.

\subsection{Channels to Obtain Information on GMF}

The public has many channels to obtain information about GMF in modern society. The results showed that $51.5 \%, 38.2 \%$, and $31.7 \%$ of the respondents got to know
GMF through TV, internet, and newspapers and magazines, respectively; in addition, some respondents obtained the information through salespersons' introduction in supermarket $(24.1 \%)$, books $(23.5 \%)$, and their relatives and friends $(17.6 \%)$. Therefore, TV, internet, and newspapers and magazines are the main approaches for consumers to obtain information.

As to the most trusted channel, $33.6 \%$ of the respondents chose relatives and friends, $31.3 \% \mathrm{TV}$, and $22.7 \%$ newspapers and magazines, while only $7.4 \%$ internet. It suggests that TV, newspapers and magazines play a significant role in guiding public opinion, while information obtained by internet is low credible.

\subsection{Risk Perception of GMF}

The safety of GMF is the bone of contention, and to date, no final decision has been reached on this matter. Consequently, consumers' opinions upon the matter may depend on the available information. Among the 238 respondents who have heard of GMF, 23.2\% considered GMF to be safe, $39.3 \%$ unsafe, and $37.5 \%$ were uncertain.

Further analysis showed that consumers who had only a little knowledge or have never heard of GMF were inclined to be uncertain about GMF safety. In contrast, consumers who were very familiar with or knew GMF tended to regard it as safe. Moreover, the number of males who deemed GMF safe was much more than that of females; the proportion of the former was $53.4 \%$, while the latter $32.3 \%$.

\subsection{Attention to GMF Label}

Due to the particularity of GMF, GMF for sale is required to put on special label in many countries. As regards the controversial matter whether GMF should put on special label, consumers' attitudes are highly consistent, because most of them hope to distinguish GMF from traditional foods by the label. In this study, up to $91.6 \%$ of the respondents wished for label. This result is similar to Ruan's [5] investigation which examined consumers' cognition of GMF label in Shenzhen.

The investigation also showed that label played an important role for consumers in choosing goods. When they were purchasing foods, $44.3 \%$ of the respondents read component description every time and $36.9 \%$ read often. Therefore, to safeguard consumers' rights and benefits, $86.1 \%$ of the respondents held the opinion that the government should adopt mandatory labeling system of GMF.

\subsection{Purchasing Intention of GMF}

To make it easier for consumers to understand, this investigation took genetically modified soybean oil, which is common in daily life, as an example to inspect consumers' purchasing intention of GMF. The result revealed that if the price of GMF was same as that of the traditional one, only $9.8 \%$ of the respondents would choose the former and $63.7 \%$ the latter; if the two prices were different, $55.0 \%$ would still buy non-GMF even though it was more expensive, while $45.0 \%$ would choose the cheaper one even though it was GMF. Hence, we can see that consumers hold prudent attitu- 
Table 1. Influence of gender and a child under 18 on consumers' risk perception level of GMF.

\begin{tabular}{|c|c|c|c|c|c|c|c|c|}
\hline \multirow{2}{*}{ Test variable } & \multicolumn{2}{|c|}{ Levene's test } & \multicolumn{4}{|c|}{ Descriptive statistical analysis } & \multicolumn{2}{|c|}{ Independent Sample t-test } \\
\hline & F-value & P-value & Grouping Variable & Number & Mean & SD & T-value & P-value \\
\hline \multirow{2}{*}{ Gender } & \multirow{2}{*}{0.003} & \multirow{2}{*}{0.354} & Male & 152 & 3.52 & 0.33 & \multirow{2}{*}{5.604} & \multirow{2}{*}{0.047} \\
\hline & & & Female & 148 & 3.94 & 0.55 & & \\
\hline \multirow{2}{*}{ Child under 18} & \multirow{2}{*}{0.160} & \multirow{2}{*}{0.289} & with & 196 & 4.12 & 0.32 & \multirow{2}{*}{-4.822} & \multirow{2}{*}{0.039} \\
\hline & & & without & 104 & 3.62 & 0.66 & & \\
\hline
\end{tabular}

Table 2. Influence of age, income, and education background on consumers' risk perception level of GMF.

\begin{tabular}{|c|c|c|c|c|c|c|c|c|}
\hline \multirow{2}{*}{ Test Variable } & \multicolumn{2}{|c|}{ Levene's Test } & \multicolumn{3}{|c|}{ Descriptive Statistical Analysis } & \multirow[b]{2}{*}{ SD } & \multicolumn{2}{|c|}{ One-way AVOVA } \\
\hline & F-value & P-value & Grouping Variable & Number & Mean & & F-value & P-value \\
\hline \multirow{3}{*}{ Age } & \multirow{3}{*}{7.354} & \multirow{3}{*}{0.058} & $<30$ & 107 & 2.47 & 0.51 & \multirow{3}{*}{3.341} & \multirow{3}{*}{0.274} \\
\hline & & & $30-55$ & 132 & 3.95 & 0.70 & & \\
\hline & & & $>55$ & 61 & 3.52 & 0.39 & & \\
\hline \multirow{3}{*}{$\begin{array}{l}\text { Personal annual } \\
\text { income }\end{array}$} & \multirow{3}{*}{0.152} & \multirow{3}{*}{0.061} & $<30000$ & 67 & 3.46 & 0.46 & \multirow{3}{*}{3.054} & \multirow{3}{*}{0.018} \\
\hline & & & $30000-60000$ & 135 & 3.98 & 0.74 & & \\
\hline & & & $>60000$ & 98 & 4.21 & 0.78 & & \\
\hline \multirow{3}{*}{$\begin{array}{l}\text { Education back- } \\
\text { ground }\end{array}$} & \multirow{3}{*}{5.973} & \multirow{3}{*}{0.547} & Low degree & 92 & 2.81 & 0.32 & \multirow{3}{*}{5.255} & \multirow{3}{*}{0.002} \\
\hline & & & Medium degree & 121 & 3.63 & 0.53 & & \\
\hline & & & High degree & 87 & 4.05 & 0.80 & & \\
\hline
\end{tabular}

de towards purchasing GMF which safety is still controversil, and they tend to buy GMF with a lower price than traditional food.

\section{INFLUENCE OF CONSUMER CHARACTERI- STICS ON GMF RISK PERCEPTION}

Taking respondents' risk perception level of GMF as dependent variable, independent sample t-test and one-way ANOVA were conducted to examine whether there were significant effects of respondent characteristics on risk perception level of GMF. The Levene's test showed that all variables conform to homoscedasticity (Tables $\mathbf{1}$ and Table 2).

Independent sample t-test was employed to determine whether consumers' gender and possession of at least one child under 18 had significant effects on risk perception of GMF. As shown in Table 1, both gender and possession of at least one child under 18 had remarkable influence on consumers' risk perception of GMF $(\mathrm{p}<0.05)$. The risk perception level of GMF was higher by females than by males. One reason for this could be that females take more responsibilities than males for food purchasing. Meanwhile, consumers with a child under 18 have remarkably higher risk perception level of GMF than those without, and it may be due to stronger requirements for food safety and nutrition out of the consideration of their children's health.

One-way ANOVA was employed to determine whether consumers' age, personal annual income and education background had significant effects on risk perception of GMF. As shown in Table 2, only education background $(p<0.01)$ and personal annual income $(p<0.05)$ influenced risk perception of GMF significantly.

The mean of risk perception level for consumers with senior high school and college degree were $3.63 \%$ and $4.05 \%$, respectively, clearly higher than those with lower degree. The possible reasons may be that the information which highly educated consumers receive is much more comprehensive, so they may obtain more negative reports about GMF to cause their higher level of risk perception.

Higher-income consumers had significantly higher risk perception level than lower-income ones. A possible reason for it may be that the former pursue higher-quality life and have higher requirement for food quality and safety, so they hold cautious attitude to GMF whose safety is still pending.5. Main factors influencing consumers' risk perception of GMF

The main factors influencing consumers' risk perceptive level of GMF were examined by 14 questions, and principal 
Table 3. Main factors influencing on consumers' risk perception of GMF.

\begin{tabular}{|c|c|c|c|c|}
\hline Index & Health Risk & Ecological Risk & Social Risk & Function Risk \\
\hline Worry about influence on human reproduction & 0.701 & 0.046 & 0.128 & 0.095 \\
\hline Worry about emergence of super-weed or super-pest & -0.142 & 0.769 & 0.095 & 0.292 \\
\hline Worry about destruction of ecological balance & 0.367 & 0.613 & 0.221 & 0.035 \\
\hline Worry about environmental pollution & 0.330 & 0.592 & 0.114 & 0.114 \\
\hline Worry about influence on food security in china & 0.400 & 0.096 & 0.879 & 0.110 \\
\hline Worry about control of breeding area by foreign countries & -0.343 & 0.055 & 0.726 & 0.255 \\
\hline Worry about inferior taste & 0.102 & 0.020 & 0.248 & 0.754 \\
\hline Eigenvalue & 2.351 & 1.871 & 1.401 & 1.027 \\
\hline Total variance explained (\%) & 35.778 & 18.392 & 12.775 & 9.315 \\
\hline Cumulative proportion of variance (\%) & 35.778 & 54.170 & 66.945 & 76.260 \\
\hline
\end{tabular}

components were extracted. First, KMO and Bartlett's test of spherical were conducted. The KMO value was 0.815 , which indicates that the 14 questions are closely related. In addition, chi-square value of Bartlett's test of spherical was $832.619(\mathrm{p}=0.000)$, which indicates that correlation matrix is significant different from identity matrix. Therefore, the data were appropriate to be analyzed by factor analysis.

Then, factor loading matrix was rotated by varimax rotation. Index 2, which indicates worry about food allergy or intoxication, was double loaded; index 12, which indicates worry about destructing natural selection, had a lower load. Therefore, these two indexes were excluded and other twelve indexes left.

On the basis of eigenvalue greater than 1, four common factors were extracted. Factor loading after rotation and four common factors were shown in Table 3. Cumulative proportion of variance of the common factors was $76.260 \%$, which indicates that these factors can adequately express the information of these indexes.

Eigenvalue of the first common factor was 2.351 and total variance explained was $35.778 \%$. It included three indexes: worry about harm to family health, children's growth and human reproduction, which can be summarized as harm of GMF to consumers' and their families' health, so it may be named as health risk.

Eigenvalue of the second common factor was 1.873 and total variance explained was $14.392 \%$. It mainly included four indexes: worry about emergence of super-weed or super-pest, destruction of biological diversity, destruction of ecological balance and environmental pollution. Because these four indexes are mainly about destruction of ecological environment, it may be named as ecological risk.

Eigenvalue of the third common factor was 1.401 and total variance explained was $10.775 \%$. It mainly included three indexes: worry about the influence on Chinese food security, control of breeding area by foreign countries and excessive intervention in biological evolution process. Thus, it may be named as social risk.

Eigenvalue of the fourth common factor was 1.211 and total variance explained was $9.315 \%$. It mainly included two indexes: worry about poor nutrition and inferior taste. Nutrition and taste are two major elements taken into consideration in purchasing food, so it may be named as function risk.

On the basis of extraction of four common factors, multivariate linear regression analysis was adopted to further inspect whether these common factors had influence on consumers' risk perception level of GMF and their influencing degree. The model is expressed as follows:

$$
Y=\beta_{0}+\beta_{1} X_{1}+\beta_{2} X_{2}+\beta_{3} X_{3}+\beta_{4} X_{4}+\varepsilon
$$

Where $Y$ refers to consumers' risk perception level of $\mathrm{GMF} ; \beta 0$ means constant term; four common factors extracted, which are health risk, ecological risk, social risk, and function risk, are independent variables $\mathrm{X} 1, \mathrm{X} 2, \mathrm{X} 3$, and $\mathrm{X} 4$; $\beta 1, \beta 2, \beta 3$, and $\beta 4$ represent unstandardized regression coefficients; $\varepsilon$ is a random disturbance term.

The results of regressive analysis showed that the adjusted R2 was $0.413, \mathrm{p}=0.000$, which indicates that the model passes test. According to Table 4, the multiple linear 
Table 4. Regression analysis of factors influencing consumers' risk perception level of GMF.

\begin{tabular}{|c|c|c|c|c|}
\hline Variable & Beta & S.E. & t & Sig. \\
\hline \hline (Constant) & 2.475 & 0.277 & 55.015 & 0.000 \\
\hline Health risk (X1) & 0.386 & 0.041 & -5.865 & 0.001 \\
\hline Ecological risk (X2) & 0.187 & 0.020 & 3.216 & 0.007 \\
\hline Social risk (X3) & 0.163 & 0.041 & 2.844 & 0.049 \\
\hline Function risk (X4) & 0.145 & 0.036 & -0.589 & 0.281 \\
\hline
\end{tabular}

regression equation that influences consumers' risk perception level of GMF can be expressed as follows:

$$
Y=2.475+0.386 X_{1}+0.187 X_{2}+0.163 X_{3}
$$

The regression equation indicates that different factors have different influencing degrees on consumers' risk perception level of GMF. Among them, health risk that consumers can perceive has the deepest influencing degree $(p<0.01)$, which conforms to reality. What consumers concern most about food is its influence on health. With the increased awareness of safety and health, consumers become prudent about GMF and tend to avoid purchasing them when faced with much negative information about GMF.

The common factor, ecological risk, also had a significant influence on consumers' risk perception of GMF $(p<0.01)$. That is, the greater destruction of transgenosis to ecological environment consumers considered, the higher risk perception level of GMF they had. This result may be related to the environmental protection idea advocated in current society. There are some opinions of harm to environment due to genetic modification, such as producing super-weed or super-pest, polluting environment and destructing ecology.

The common factor, social risk, also had a significant influence on consumers' risk perception of GMF $(p<0.05)$. On the one hand, consumers worry that spreading GMF will cause Chinese breeding area under the control of transnational corporations with dominant position in genetically modified technology development and commercial operation, which will pose threat to native food security. On the other hand, to change organism's biological features, transfer some gene from one species to another may affect normal evolution process of plants. This also contributes to consumers' worry about GMF to some extent, and then intensifying their risk perception of GMF.

By contrast, the common factor, function risk, had no significant influence on consumers' risk perception of GMF. Compared to food nutrition and taste, food safety is much more important for the public. For newborn high-tech GMF, consumers pay more attention to its safety. For example, whether it will give rise to food allergy or intoxication. Meanwhile, for vegetarians or religious persons, the most important isn't its nutrition or taste, but its accordance with doctrines and ethics. Consequently, compared with the safety and ethics of GMF, its food functions such as nutrition and taste have less influence on consumers' risk perception of GMF. This result is similar to that of Dong [3].

\section{RESEARCH CONCLUSIONS AND POLICY SUG- GESTIONS}

Focusing on consumers' risk perception of GMF and its influencing factors in Jiangsu province, this paper comes to the following conclusions.

First, although up to $82.3 \%$ of the respondents have heard of GMF, their congnition of GMF is vague. Consumers gain information about GMF mainly by TV, internet, and newspapers and magazines, but they most distrust the information from internet. Moreover, they most trust the information from friends and relatives.

Second, most consumers worry about GMF safety and they hope the government set up mandatory labeling system of GMF to help them to differentiate GMF from traditional food.

Third, consumers are more likely to buy GMF with a lower price than traditional food. If there is no difference in price, most consumers are reluctant to buy GMF.

Fourth, some characteristics of consumer, including age, education background, personal annual income, and whether has a child under 18 , have significant influence on their risk perception of GMF. Females and those with children under 18 have obviously higher level of risk perception of GMF than males and those without children under 18. Furthermore, compared with consumers with lower education degree or lower income, higher-educated or higher-income consumers have much higher level of risk perception of GMF.

Fifth, different factors impose different influencing degrees on consumers' risk perception of GMF. According to influencing degree, they can be listed from the highest to the lowest as health risk (0.368), ecological risk (0.187) and social risk $(0.163)$. However, function risk has no significant influence on consumers' risk perception of GMF.

According to the conclusions, policy suggestions can be summarized as follows.

First, the government should strengthen the propaganda of genetically modified technology as well as GMF through 
$\mathrm{TV}$, newspapers and magazines, and ensure that the information access to consumers is scientific and understandable. Popularization of the knowledge of GMF may help the consumers get rid of confusion about GMF, so may effectively reduce consumers' risk perception of GMF.

Second, the government should perfect supervision policy on GMF and enhance consumer confidence in GMF. Because GMF label plays an important part in consumers' purchasing decision, the government need to reinforce standardized management of GMF label and supervision of enterprises that manufacture GMF. Meanwhile, the government should guide consumers to have a correct understanding of GMF and its labels, thus guarantee consumer right to the truth and the options sufficiently, which is conducive to improving consumer welfare.

\section{CONFLICT OF INTEREST}

The author confirms that this article content has no conflict of interest.

\section{ACKNOWLEDGEMENTS}

The authors are grateful for the financial support of the social science project of Jiangsu Education Department of China No. 2013SJD630063, Yangzhou university humanity and social science project No. xjj2014-67, Key Projects of National Social Science Foundation of China No. 14ZDA069, the National Natural Science Foundation of China No. 71273117, Central University Basic Research Funds No. JUSRP51325A \& JUSRP51416B, the Project of the Six Top Talents in Jiangsu Province No. 2012-JY-002 and the project of college Innovation Team of Jiangsu Province social science No. 2013-011, Soft Science Research Project of Yangzhou No. YZ2014250.

\section{REFERENCES}

[1] S. Liu, J. Huang, and J. Bai, "Consumer's willingness to pay genetically modified foods in China", Journal of International Food and Agribusiness Marketing, vol. 43, pp. 571-584, 2006.

[2] J. Scully, "Genetic engineering and perceived levels of risk", British Food Journal, vol. 105, no. 1-2, pp. 59-77, 2003.

[3] Y. Y. Dong, Z. H. Qi, and D. M. Zhang, "Effect of perceived risks of genetically modified food on consumers' purchase intention: Based on a survey conducted in Wuhan", Journal of China Agricultural University, vol. 19, no. 3, pp. 27-33, 2014.

[4] F. N. Zhong and Y. L. Ding, "Consumer awareness and response to genetically modified food in Nanjing”, China Rural Survey, no. 1, pp. 22-27, 2004.

[5] J. L. Ruan, C. Chen, and L. H. Chen, "Investigation and analysis of consumer recognition of genetically modified foods and transgenic labelling: a case study of Shenzhen city", Modern Food Science and Technology, vol. 29, no. 4, pp. 848-852, 2013.

(C) Qin et al.; Licensee Bentham Open.

This is an open access article licensed under the terms of the Creative Commons Attribution Non-Commercial License (http://creativecommons.org/licenses/by-nc/3.0/) which permits unrestricted, non-commercial use, distribution and reproduction in any medium, provided the work is properly cited. 